\title{
Massive aggrecan and versican accumulation in thoracic aortic aneurysm and dissection
}

Frank S. Cikach, ${ }^{1}$ Christopher D. Koch, ${ }^{2,3}$ Timothy J. Mead, ${ }^{2}$ Josephine Galatioto, ${ }^{4}$ Belinda B. Willard, ${ }^{5}$ Kelly B. Emerton, ${ }^{6}$ Matthew J. Eagleton, ${ }^{7}$ Eugene H. Blackstone, ${ }^{8}$ Francesco Ramirez, ${ }^{4}$

Eric E. Roselli, ${ }^{8,9}$ and Suneel S. Apte ${ }^{2}$

${ }^{1}$ Cleveland Clinic Lerner College of Medicine of Case Western Reserve University, Cleveland, Ohio, USA. ${ }^{2}$ Department of Biomedical Engineering, Cleveland Clinic Lerner Research Institute, Cleveland, Ohio, USA. ${ }^{3}$ Department of Chemistry, Cleveland State University, Cleveland, Ohio, USA. ${ }^{4}$ Department of Pharmacological Sciences, Icahn School of Medicine at Mount Sinai, New York, New York, USA. ${ }^{5}$ Proteomics and Metabolomics Core, Cleveland Clinic Lerner Research Institute, Cleveland, Ohio, USA. ${ }^{6}$ Cleveland Clinic Innovations, ${ }^{7}$ Department of Vascular Surgery, ${ }^{8}$ Department of Thoracic and Cardiovascular Surgery, and ${ }^{9}$ Aorta Center, Cleveland Clinic, Cleveland, Ohio, USA.

Proteoglycan accumulation is a hallmark of medial degeneration in thoracic aortic aneurysm and dissection (TAAD). Here, we defined the aortic proteoglycanome using mass spectrometry, and based on the findings, investigated the large aggregating proteoglycans aggrecan and versican in human ascending TAAD and a mouse model of severe Marfan syndrome. The aortic proteoglycanome comprises 20 proteoglycans including aggrecan and versican. Antibodies against these proteoglycans intensely stained medial degeneration lesions in TAAD, contrasting with modest intralamellar staining in controls. Aggrecan, but not versican, was increased in longitudinal analysis of $F b n 7^{\mathrm{mgR} / \mathrm{mgR}}$ aortas. TAAD and $F b n 7^{\mathrm{mgR} / \mathrm{mgR}}$ aortas had increased aggrecan and versican mRNAs, and reduced expression of a key proteoglycanase gene, ADAMTS5, was seen in TAAD. $F b n 7^{\mathrm{mgR} / \mathrm{mgR}}$ mice with ascending aortic dissection and/or rupture had dramatically increased aggrecan staining compared with mice without these complications. Thus, aggrecan and versican accumulation in ascending TAAD occurs via increased synthesis and/or reduced proteolytic turnover, and correlates with aortic dissection/rupture in Fbn $7^{\mathrm{mgR} / \mathrm{mgR}}$ mice. Tissue swelling imposed by aggrecan and versican is proposed to be profoundly deleterious to aortic wall mechanics and smooth muscle cell homeostasis, predisposing to type-A dissections. These proteoglycans provide potential biomarkers for refined risk stratification and timing of elective aortic aneurysm repair.

Authorship note: FSC and CDK contributed equally as co-first authors.

Conflict of interest: EER is a clinical investigator and consultant and received lecture honoraria from W.L. Gore.

Submitted: August 30, 2017

Accepted: January 30, 2018

Published: March 8, 2018

Reference information: JCI Insight. 2018;3(5):e97167. https:// doi.org/10.1172/jci.insight.97167.

\section{Introduction}

Thoracic aortic aneurysm and dissection (TAAD) causes significant morbidity and mortality, yet the molecular changes occurring in the aortic wall that promote dilatation and predisposition to dissection are poorly understood. Gene mutations affecting components of the vascular smooth muscle cell (SMC) contractile apparatus such as smooth muscle $\alpha$-actin as well as extracellular matrix (ECM) components, such as fibrillin-1 (leading to Marfan syndrome) result in TAAD, implying that a continuum from cells to ECM is required for maintenance of aortic integrity (1-4). Intriguingly, the histopathologic hallmark of TAAD, termed medial degeneration, is a shared characteristic of these diverse conditions (5). Proteoglycan accumulation, typically ascertained by Alcian blue staining, along with elastic fiber fragmentation and SMC loss, is a defining component of medial degeneration $(5,6)$. However, the molecular identity of the proteoglycans accumulating in TAAD and their significance in TAAD pathogenesis remain undefined. Under normal conditions, proteoglycans such as versican have been shown to contribute to the transmural distribution of residual stresses in the ascending aorta, and normalize tensile stresses across its wall (7). However, recent work utilizing computational models suggests that pathologic accumulation of versican in the aortic wall can generate an interstitial swelling pressure that may be mechanically disruptive to fibrillar ECM and may initiate delamination in the tunica media (6, 8-11). Proteoglycans can generate a Donnan osmotic pressure due to inherent fixed negative charges on glycosaminoglycan (GAG) side chains $(12,13)$. These fixed charges attract mobile counterions such as sodium and thus, interstitial water, driv- 
ing a swelling pressure in the region carrying the fixed charges. Computational models that have incorporated the effects of proteoglycan pools into an otherwise normal aortic media have predicted that these pools result in increased stresses of magnitude sufficient to sever elastic fibers and microfibril-cell connections $(9,10)$. Furthermore, it was predicted that asymmetric proteoglycan/GAG pools, i.e., with complex shapes, coalescing pools, or finger-like projections into the media or higher fixed-charge density would result in greater local stress concentrations (9). In addition to contributing to medial delamination, these swollen aggregates of proteoglycans may alter the tension placed on microfibril-cell connections that are vital to mechanosensing of aortic SMCs (9). This modification to the mechanotransduction apparatus may subvert the normal signaling required for aortic SMCs to sense and respond to stress, contributing to dysregulated ECM homeostasis $(8-10,14,15)$.

Although previous proteomic analyses of the aorta identified several proteoglycans (16), one aim of the present study was to selectively isolate the proteoglycans in the ascending aortas of normal and TAAD patients for characterization of their complete proteoglycanomes. This analysis identified not only versican, but also aggrecan, a large aggregating proteoglycan with considerably greater potential for mechanical and cellular disruption than versican. We therefore determined the spatial distribution of aggrecan and versican in normal and ascending TAAD aortas. We also sought to understand the mechanism of their accumulation by investigating the expression of the genes and proteases implicated in their turnover. Finally, we directly determined the relationship between proteoglycan accumulation and ascending aortic dissection or rupture in a mouse model of severe Marfan syndrome. The findings suggest that accumulation of aggregating proteoglycans, particularly aggrecan, within the aortic tunica media may be profoundly disruptive to ECM integrity, potentially contributing to the pathogenesis of thoracic aortic aneurysm and dissection.

\section{Results}

Aggrecan and versican are components of the normal and TAAD proteoglycanomes. Proteoglycan isolation from human ascending aorta by anion-exchange chromatography (Figure 1A) was confirmed by the detection of chondroitin sulfate (CS) in high-salt fractions using safranin-O dot blot, fluorophore-assisted carbohydrate electrophoresis (FACE), and versican Western blot (Figure 1, B-D; see complete unedited blots in the supplemental material). Liquid chromatography-tandem mass spectrometry (LC-MS/MS) of isolated proteoglycans from 3 control and 3 TAAD cases identified a total of 20 proteoglycans known to bear GAG species, i.e., CS, dermatan sulfate, heparan sulfate (HS), and keratan sulfate (Table 1). Of these, the large hyaluronan-binding aggregating proteoglycans aggrecan and versican, small leucine-rich CS proteoglycans decorin and biglycan, and HS proteoglycans perlecan and collagen XVIII were identified in all 6 proteoglycanomes, whereas others were identified inconsistently (Table 1). Identification of aggrecan and versican in normal aorta and TAAD proteoglycanomes, together with their propensity for generating significant swelling pressure when confined in tissue, led to their prioritization for follow-up investigation in normal and TAAD samples.

Aggrecan and versican accumulate in the tunica media and medial degeneration lesions in ascending TAAD. Movat pentachrome stains of normal aortas showed a typical lamellar structure, whereas TAAD aortas consistently revealed medial degeneration with areas of intense blue staining indicating proteoglycan accumulation, along with a sparse SMC population and loss of elastic lamellae with fragmented elastic fibers (Supplemental Figure 1; supplemental material available online with this article; https://doi.org/10.1172/ jci.insight.97167DS1). In control subjects, aggrecan immunofluorescence revealed varying patterns, ranging from sparse staining confined to the pericellular matrix of a subpopulation of aortic SMCs to a multifocal intralamellar distribution in the tunica media (Figure 2A and Supplemental Figure 2A). However, TAAD aortas consistently demonstrated stronger aggrecan staining restricted to the tunica media that was not only intra- and translamellar, but was intense in medial degeneration lesions (Figure 2, A and B, and Supplemental Figure 2A). Versican immunofluorescence in control aortas revealed a uniform intralamellar distribution with intact medial architecture (Figure 2A and Supplemental Figure 2B). In contrast, TAADs demonstrated increased versican staining that spanned multiple lamellar units and was intense in areas of medial degeneration (Figure 2, A and B, and Supplemental Figure 2B). Versican staining intensity was greater in TAAD aortas than controls (Figure 2A and Supplemental Figure 2B). Combined immunofluorescence for aggrecan and versican showed that some areas of medial degeneration contained aggrecan, others contained versican, and some contained both (Figure 2A). Aggrecan and versican staining was consistently stronger in TAAD patients independent of the primary etiology (Supplemental Figure 2, A and B). Because aggrecan accumulation was unexpected, it was confirmed using another aggrecan-specific antibody, with similar results (Supplemental Figure 3). 
A
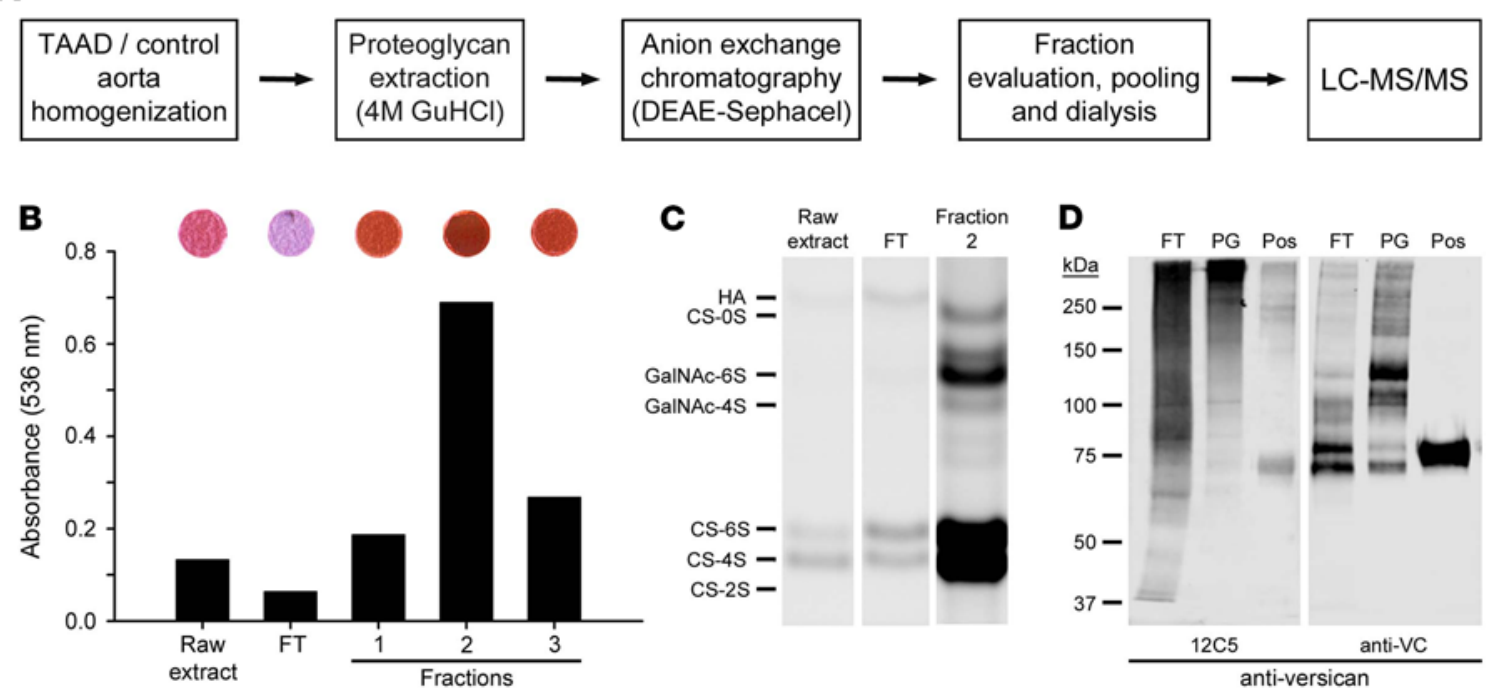

Figure 1. Isolation and analysis of proteoglycans from human control ascending aortas and ascending thoracic aortic aneurysm and dissection (TAAD). (A) Workflow of human aorta proteoglycan (PG) extraction, isolation by diethylaminoethyl (DEAE)-Sephacel chromatography and analysis. LC-MS/MS, liquid chromatography-tandem mass spectrometry; GuHCl, guanidine hydrochloride. (B) Top: Evaluation of DEAE-Sephacel fractions for glycosaminoglycan (CAG) content by safranin-O dot-blot assay. Bottom: Colorimetric quantification of safranin- 0 dot blot after solubilization of precipitate using cetylpyridinium chloride. FT, flow-through. (C) Fluorophore-assisted carbohydrate electrophoresis analysis for hyaluronan (HA) and chondroitin sulfate (CS) GAGs. A preparation of purified HA and CS mono- and disaccharides was used as the standard as indicated on the left. GaINAc, N-acetylgalactosamine. Sulfation positions of the saccharides are indicated as non-sulfated (OS), 2S, 4S, and 6S. (D) Western blot analysis of FT and pooled DEAE-Sephacel PG-containing fractions 1-3. Monoclonal antibody $12 \mathrm{C} 5$ recognizes the versican $\mathrm{C} 1$ domain and polyclonal antibody VC recognizes both versican and a disintegrin-like and metalloprotease domain with thrombospondin type 1 motifs (ADAMTS) protease-cleaved versican. 12C5 Western blot was performed under nonreducing conditions and VC Western blot under reducing conditions. All samples were digested with chondroitinase ABC prior to electrophoresis. Medium from HEK 293T cells transiently expressing recombinant human versican $\mathrm{N}$-terminal fragment (versikine) was used as the positive control (Pos) for both antibodies.

The aggrecan neoepitope antibody revealed a variable distribution of cleaved aggrecan in control aorta ranging from staining of pericellular matrix of a minority of vascular SMCs (Figure 3) to staining that spanned lamellae. Control aortas had minimal intralamellar staining with the versican neoepitope antibody, whereas TAADs showed cleaved versican in areas of medial degeneration (Figure 3). While versican cleavage in TAAD aortas often colocalized with versican deposition, indicating a relatively uniform turnover, the larger aggrecan pools were devoid of cleaved aggrecan (note absence of yellow staining indicating colocalization in top row of Figure 3), which had mostly a pericellular localization.

Aggrecan, but not versican, accumulates in a mouse model of severe Marfan syndrome. Proteoglycan dynamics was not previously studied in mouse models of aortic disease. The temporal relationship of aggrecan and versican with ascending aortic aneurysm progression and normal aortic growth was therefore compared in a longitudinal analysis of $F b n I^{\mathrm{mgR} / \mathrm{mgR}}$ mice, which are widely used as a model of severe Marfan syndrome (17) and wild-type littermates. $F b n 1^{\mathrm{mgR} / \mathrm{mgR}}$ mice underexpress fibrillin-1 and die from TAAD within the first few months of postnatal life (average survival, 2.5 months) (18). Immunofluorescence analysis delineated the spatial distribution of aggrecan within the healthy and diseased aortas. At postnatal day 16 (P16), aggrecan was confined to the inner third of the wild-type tunica media and limited to one-half of the circumference of the aorta, whereas $F b n I^{\mathrm{mgR} / \mathrm{mgR}}$ mice had more intense aggrecan staining throughout the aortic circumference. Nearly all aggrecan staining seen at P16 in wild-type aorta was lost by P30, and was absent at P45, P60, and P90 (Figure 4A). In contrast, P30, P45, P60, and P90 Fbn $1^{\mathrm{mgR} /}$ $\mathrm{mgR}$ aorta consistently demonstrated aggrecan staining, which was significantly increased as determined from measured mean integrated density (median [interquartile range (IQR)]): at P30, (6.0 [37.3] Fbn $1^{\mathrm{mgR} /}$ ${ }^{\mathrm{mgR}}$ vs. 0 [0] wild type, $\left.P=0.034\right)$; at $\mathrm{P} 45$ (34.9 [35.4] Fbn $1^{\mathrm{mgR} / \mathrm{mgR}}$ vs. 0 [0.3] wild type, $\left.P=0.034\right)$; and P60 (1.5 [2.3] $F b n 1^{\mathrm{mgR} / \mathrm{mgR}}$ vs. 0 [0.2] wild type, $\left.P=0.05\right)$. Thus, the observed difference in aggrecan staining was most conspicuous at P45 (Figure 4A). Robust cleaved aggrecan staining was evident in the wild-type aorta. It increased from P16 to P60, and was slightly reduced at P90, but was absent at P45 (Figure 4B). 
Table 1. Proteoglycanomes of normal and ascending TAAD aortas

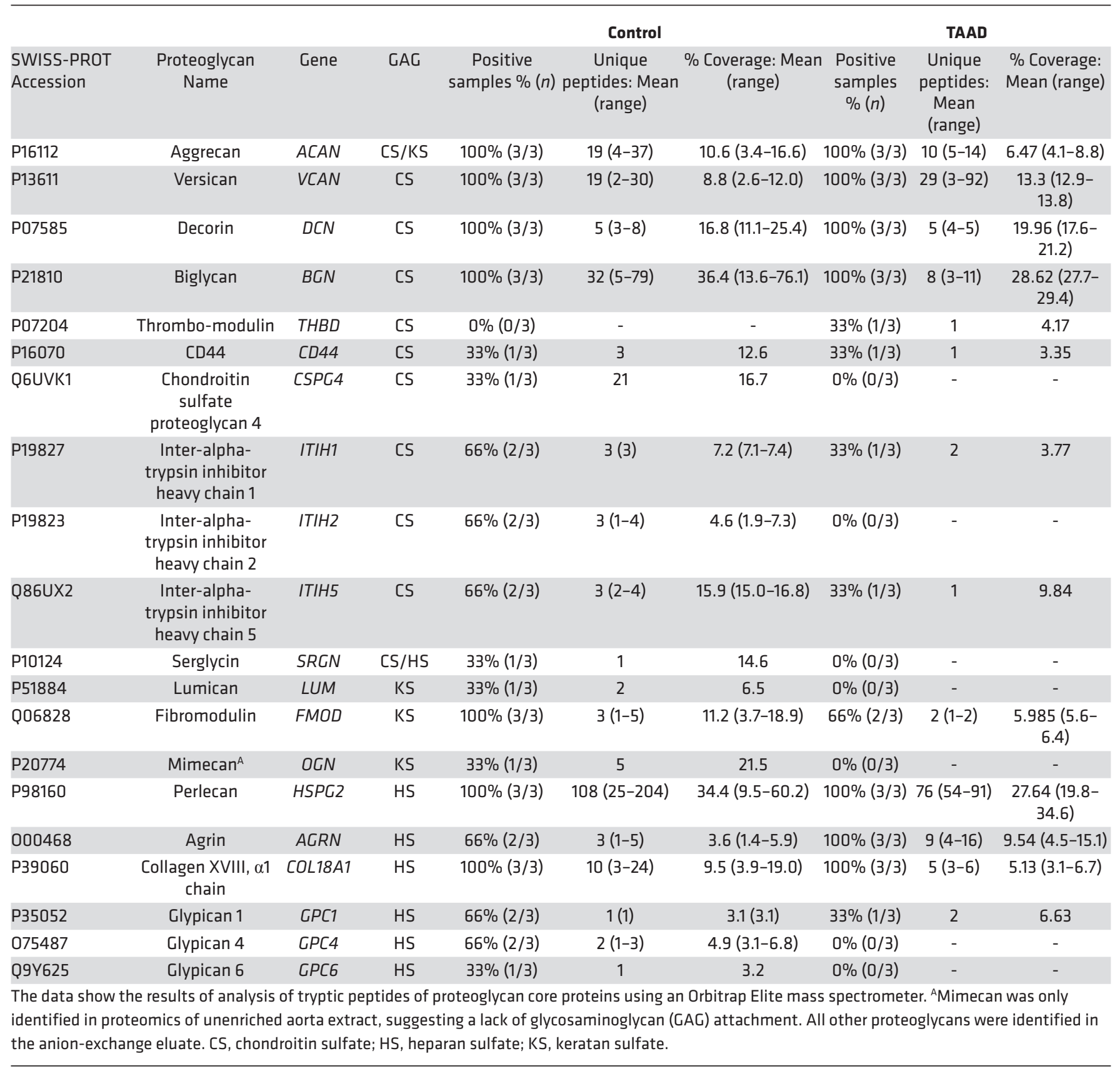

In contrast, $F b n 1^{\mathrm{mgR} / \mathrm{mgR}}$ aortas had little cleaved aggrecan in the aortas at all time points examined other than P16, with a significant difference at P60 (1.9 [1.8] vs. 26.2 [4.0], $P=0.05$ ) (Figure 4B). We observed little to no versican nor cleaved versican immunofluorescence in most wild-type and $F b n I^{\mathrm{mgR} / \mathrm{mgR}}$ aortas at the time points examined (Supplemental Figure 4).

Fbn $1^{m g R / m g R}$ ascending aortas that dissect or rupture have consistently greater aggrecan accumulation than ascending aortas of euthanized Fbn $1^{\mathrm{mgR} / \mathrm{mgR}}$ mice. A reduction in aggrecan staining was noted in $F b n 1^{\mathrm{mgR} /}$ ${ }^{\mathrm{mgR}}$ mice after P45 (Figure 4, A and B). Therefore, we stained ascending aortas of $F b n 1^{\mathrm{mgR} / \mathrm{mgR}}$ mice that died of aortic dissection/rupture for aggrecan and quantitatively compared this to ascending aortas of $F b n I^{\mathrm{mgR} / \mathrm{mgR}}$ mice that were euthanized at similar time points (Figure 5). This analysis revealed a dramatic increase in aggrecan accumulation in aortas from mice that died of aortic dissection/rupture versus euthanized mice. In these mice, aggrecan staining spanned the full thickness and entire circumference of 
A
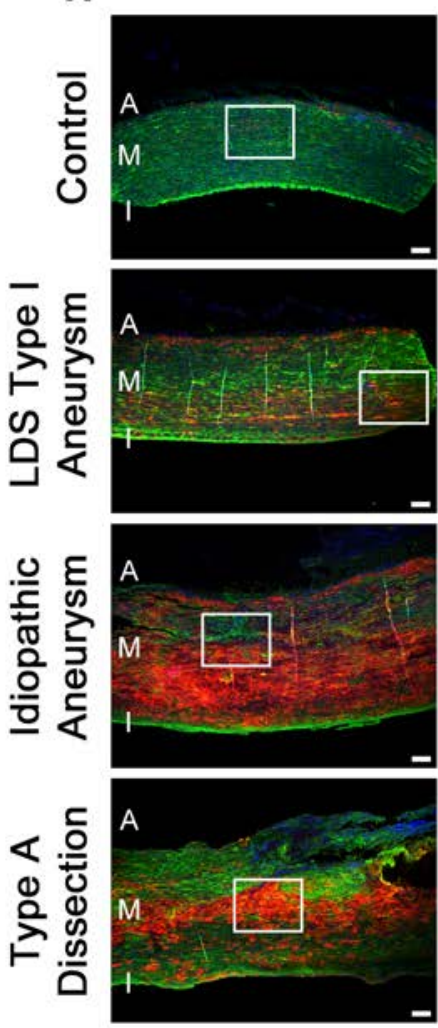

B
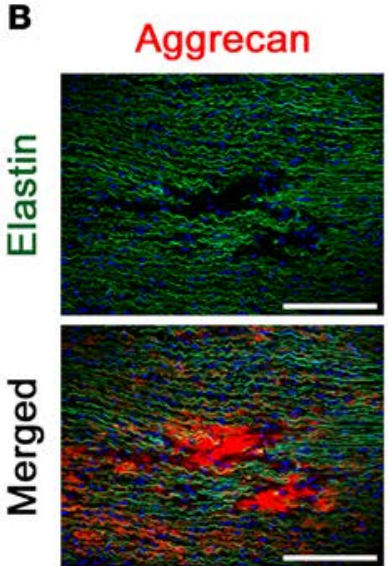

Aggrecan
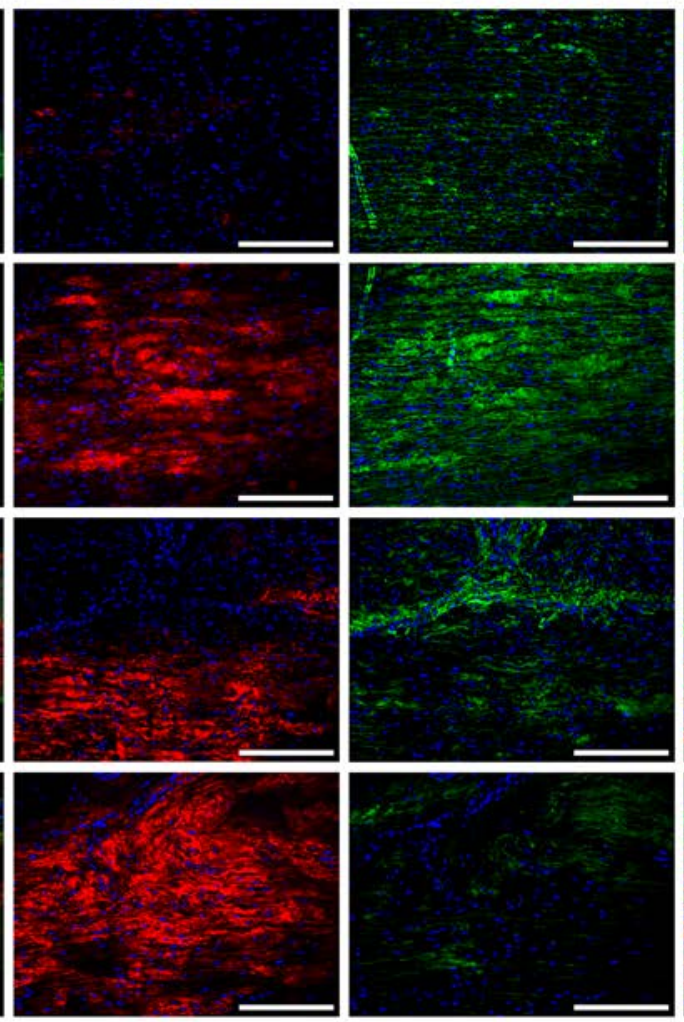

Versican
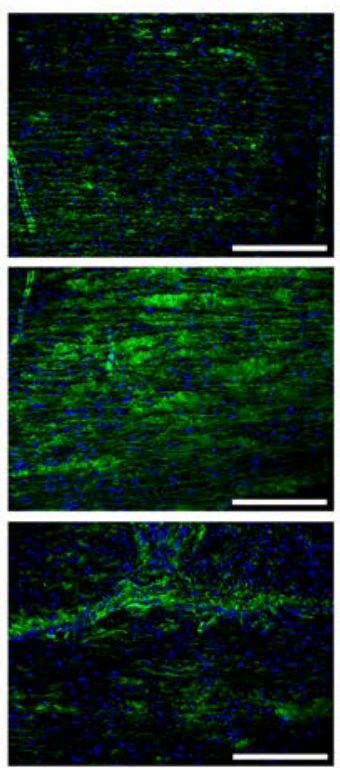

Merged
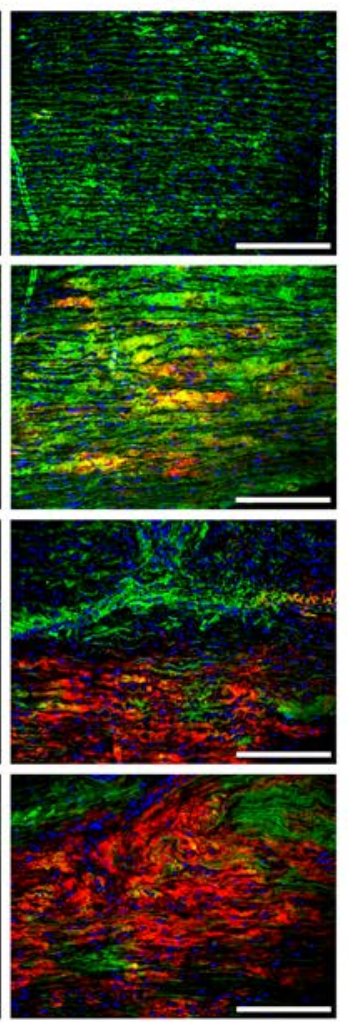

C

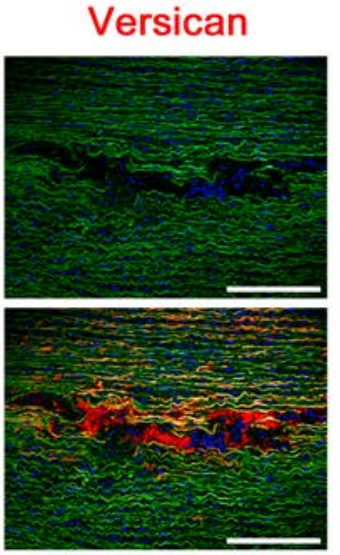

Figure 2. Aggrecan and versican accumulate in human ascending thoracic aortic aneurysm and dissection (TAAD). (A) Aggrecan (red) and versican (green) accumulate primarily within the aortic tunica media (M). A and I indicate the aortic adventitia and intima, respectively. Images in columns 2-4 represent higher magnification views of the areas contained within the white boxes in the left-hand column. LDS, Loeys-Dietz syndrome. Scale bars: $200 \mu \mathrm{m}$. The images are representative of 6 control and 20 TAAD cases. (B) Intense aggrecan and versican staining is seen in areas of medial degeneration in a case of idiopathic TAAD (left-hand panels) and Marfan syndrome (right-hand panels), identified here as regions with fragmented or absent elastic fibers (green) and few smooth muscle cells (nuclei stained blue) in TAAD aortas. The images are representative of 20 TAAD cases. Scale bars: $200 \mu \mathrm{m}$. (C) Quantification of aggrecan and versican within medial degeneration lesions. Representative areas of aggrecan and versican staining were quantified for control samples. Each data point represents an independent biological replicate. Differences between groups were assessed using the Mann-Whitney U test with JMP Pro software (version 13). Bars represent the median with interquartile range. Aggrecan: $n=4$ control, $n=19$ TAAD. Versican: $n=4$ control, $n=20$ TAAD. ${ }^{* *} P \leq 0.01$.

the tunica media of the ascending aorta, including strong staining in the areas of dissection and rupture (Figure 5, A and B). There was an approximately 30-fold increase in aggrecan staining in post-P45 Fbn$I^{\mathrm{mgR} / \mathrm{mgR}}$ mice that died of aortic dissection/rupture versus post-P45 euthanized $F b n I^{\mathrm{mgR} / \mathrm{mgR}}$ mice $(34.6$ [15.4] dissection/rupture vs. 1.2 [3.3] euthanized, $P=0.006$ ) (Figure 5C). 
Control

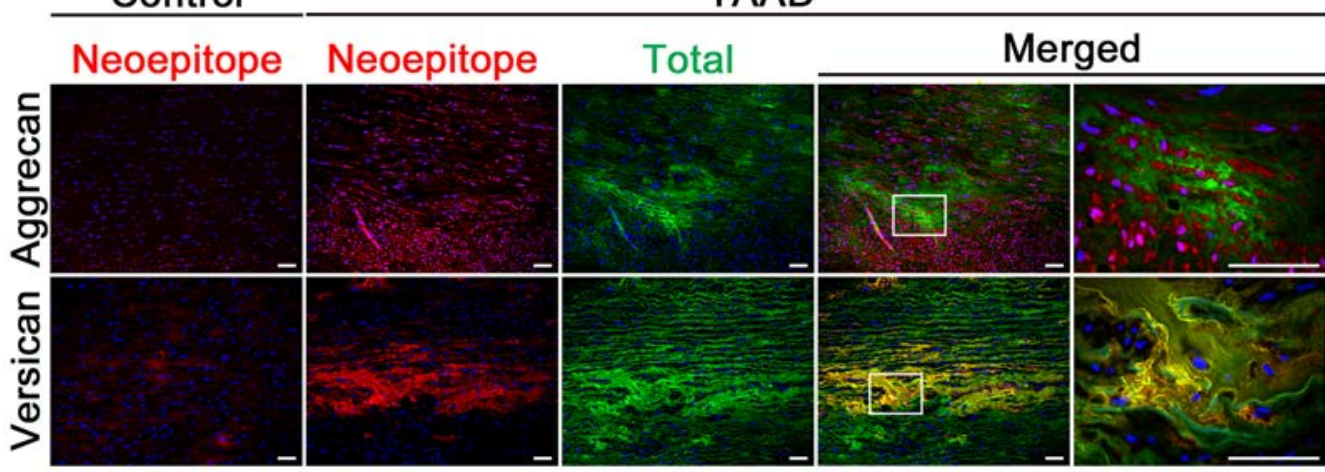

Figure 3. A disintegrin-like and metalloprotease domain with thrombospondin type 1 motifs (ADAMTS) protease-mediated aggrecan and versican proteolysis occurs in human ascending thoracic aorta aneurysm and dissection (TAAD). Representative immunofluorescence images demonstrate the distribution of ADAMTS-generated aggrecan (anti-NITECE) and versican (anti-DPEAAE) neoepitopes in a control subject (left-hand column) and a TAAD subject having Marfan syndrome. Minimal staining for both proteoglycan neoepitopes is evident in the control. The TAAD aorta shows intense staining for cleaved versican and relatively lower staining intensity for cleaved aggrecan in areas of medial degeneration. The right-hand (merged) column shows a higher-magnification view of the area contained within white boxes in the left (merged) column. The images shown are representative of 6 controls and 20 TAAD cases. Scale bars: $50 \mu \mathrm{m}$.

Increased proteoglycan expression and reduced ADAMTS5 $m R N A$ in TAAD aortas. To determine the potential mechanisms of the observed accumulation of aggrecan and versican in TAAD, we used real-time quantitative PCR (RT-qPCR) to evaluate expression of $A C A N$ and $V C A N$ and the genes encoding proteases that have been implicated in their turnover, namely ADAMTS1, ADAMTS4, ADAMTS5, ADAMTS9, ADAMTS15, and ADAMTS20. This analysis revealed a significant increase in $V C A N$ but not $A C A N$ expression in TAAD (Supplemental Figure 5). Notably, ADAMTS5 mRNA, encoding a disintegrin-like and metalloprotease domain with thrombospondin type 1 motifs 5 (ADAMTS5), a protease with a major role in aggrecan and versican turnover $(19,20)$, was significantly reduced in TAAD aortas (Supplemental Figure 5). Expression of other ADAMTS protease genes was not significantly altered.

$A C A N$ in situ hybridization in control human aorta demonstrated sporadic expression by a few SMCs (Figure 6A). In contrast, numerous SMCs in TAAD aortas expressed $A C A N$, with clusters of $A C A N$-positive cells typically seen in medial degeneration lesions (Figure 6A). In situ hybridization of $V C A N$ in control human aorta demonstrated expression by most cells in the inner third of the tunica media, with reduced staining in the outer medial layers (Figure 6A). In contrast, TAADs demonstrated intense expression in the inner third of the tunica media with persistent $V C A N$ expression in the outer tunica media (Figure 6A). Acan expression was sparse in wild-type mouse aorta at P16 and P45 (Figure 6B). In striking contrast, Acan was expressed by most SMCs in Fbn $1^{\mathrm{mgR} / \mathrm{mgR}}$ aortas at P16 and P45 (Figure 6B). Wild-type and Fbn $1^{\mathrm{mgR} / \mathrm{mgR}}$ mouse aortas at P16 and P45 displayed limited Vcan expression, restricted to scattered cells within the tunica media (Figure 6B). Transcriptome comparison of the $F b n 1^{\mathrm{mgR} / \mathrm{mgR}}$ aorta and wild-type aorta ranked Acan at or near the top of matrix-related genes that are dysregulated from early (P16, P30) to a more advanced stage (P60) of TAAD, whereas Vcan was not as substantially altered (Supplemental Table 4).

\section{Discussion}

In order to define the proteoglycanomes of the normal and TAAD aortas, we reduced the molecular complexity of aortic extracts by selectively isolating proteoglycans for proteomic analysis. We consistently identified aggrecan, versican, and 4 other proteoglycans among a total of 20 core proteins. Because aggrecan and versican form aggregates with considerable potential for tissue disruption, we investigated their distribution in TAAD and identified dramatic accumulation, especially in medial degeneration lesions, strongly suggestive of altered biosynthesis and/or proteolytic turnover. RNA analyses supported increased $A C A N$ and $V C A N$ transcription in human TAAD and increased Acan mRNA in mouse TAAD. ADAMTS proteases, a group of secreted proteolytic enzymes, have been implicated as major aggrecanases and versicanases $(20,21)$. Although staining for aggrecan and versican neoepitopes suggests ongoing proteolysis by ADAMTS proteases in TAAD aortas, we also observed reduced expression of ADAMTS5, a key protease 
A

P16

P30

P45

P60

P90
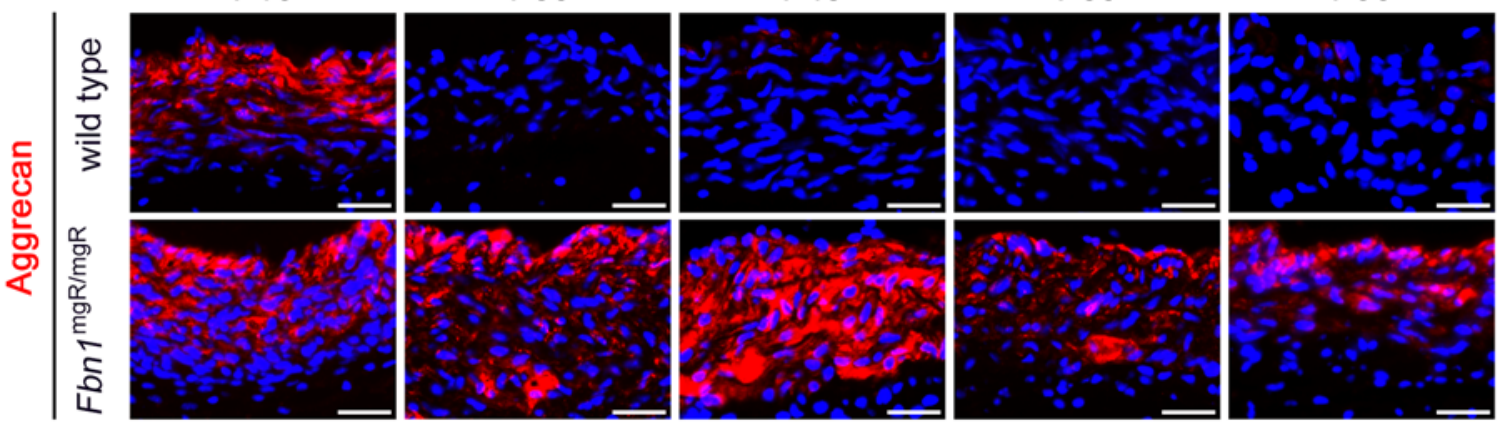

B
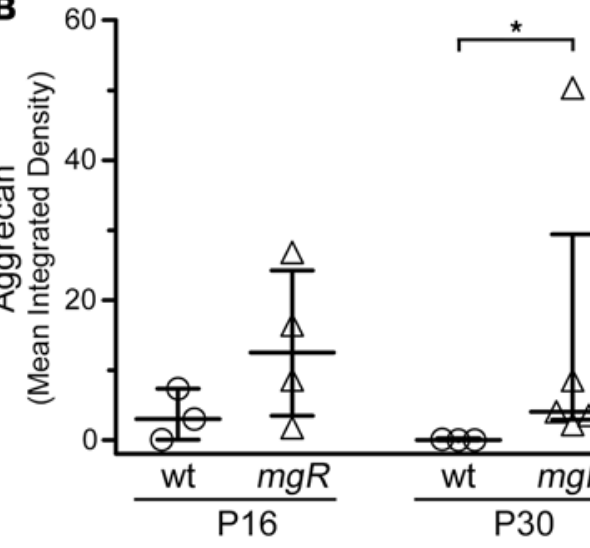

C
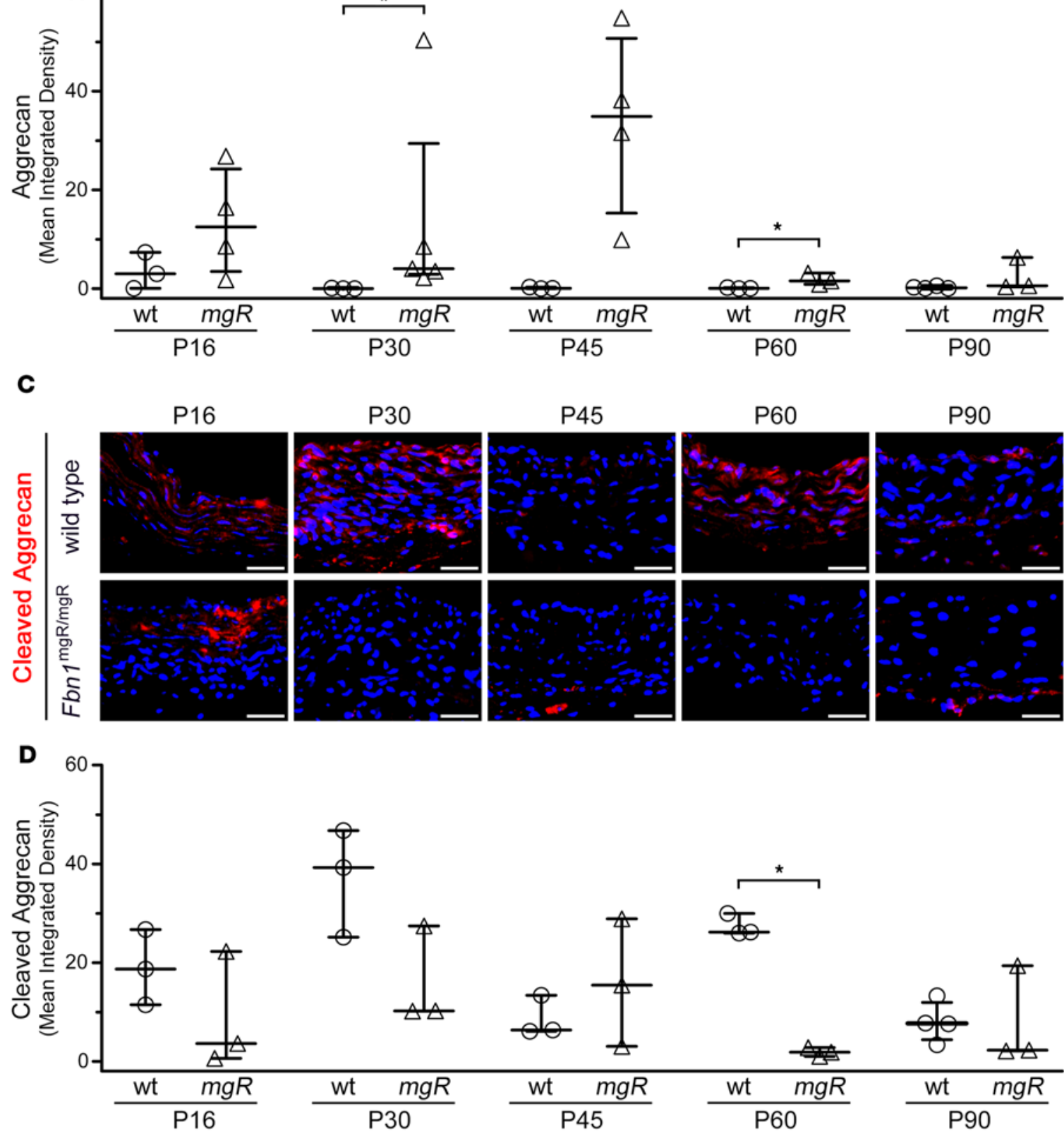

$\triangle$

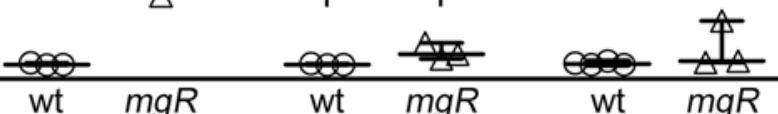
P45 
Figure 4. Aggrecan accumulates in the $\mathbf{F b n} \mathbf{7 g R / m g R}^{\mathrm{mg}}$ ascending aorta. The temporal and spatial distribution of aggrecan and a disintegrin-like and metalloprotease domain with thrombospondin type 1 motifs (ADAMTS) protease-cleaved aggrecan was determined in wild-type mice and Fbn ${ }^{\mathrm{mgR} / \mathrm{mgR}}$ mice, a model for severe Marfan syndrome, at various postnatal (P) ages (shown in days). (A) Aggrecan immunofluorescence in wild-type and Fbn $1^{\mathrm{mgR} / \mathrm{mgR}}$ aortas. Images are representative of staining in $n=3$ wild type and $n=3$ or 4 (PgO) at each time point for Fbn ${ }^{\mathrm{mgR} / \mathrm{mgR}}$ mice. Scale bars: $100 \mu \mathrm{m}$. (B) Quantification of fluorescence signal in the wild-type (wt) and $F b n 1^{\mathrm{mgR} / \mathrm{mgR}}(\mathrm{mgR})$ groups represented in $\mathbf{A}$ are shown as median with interquartile range. Differences between groups were assessed using the Mann-Whitney $U$ test with JMP Pro software (version 13 ). $n=3-4 .{ }^{*} P \leq 0.05$. (C) Immunofluorescence of the NITEGE neoepitope arising from ADAMTS-cleaved aggrecan in wild-type and Fbn $7^{\mathrm{mgR} / \mathrm{mgR}}$ aortas. Images representative of staining in $n=3$ or 4 (Pgo) wildtype and $n=3$ at each time point for Fbn $1^{\mathrm{mgR} / \mathrm{mgR}}$ mice. Scale bars: $100 \mu \mathrm{m}$. (D) Quantification of fluorescence signal in the experimental groups represented in C shown as median with interquartile range. $n=3-4 .{ }^{*} P \leq 0.05$.

in their turnover. In contrast, ADAMTS1 and ADAMTS4 mRNAs, encoding major proteoglycan-degrading enzymes (20), were not significantly altered. These findings suggest a role for increased production of aggrecan and versican, as well as reduced proteolytic turnover over an extended period of time as the basis for a net accumulation of these proteoglycans in TAAD.

Since a shortcoming of human postoperative specimen analysis is lack of temporal information, we conducted a longitudinal analysis of aggrecan and versican content, gene expression, and proteolytic turnover in a mouse model of severe Marfan syndrome having early onset, progressive ascending thoracic aortic aneurysms, and lethal thoracic aortic dissection/rupture (18). The results were concordant with the major findings in human TAAD in most respects, including aggrecan accumulation, increased Acan mRNA expression, and reduced aggrecan proteolysis relative to wild-type mice. A notable contrast with human TAAD was that versican was present at low levels in wild-type and $F b n 1^{\mathrm{mgR} / \mathrm{mgR}}$ mouse aortas. In situ hybridization identified dramatically increased Acan mRNA in $F b n 1^{\mathrm{mgR} / \mathrm{mgR}}$ tunica media SMCs, although Vcan mRNA expression was modestly increased. Extrapolating the longitudinal analysis of aggrecan and versican dynamics in $F b n 1^{\mathrm{mgR} / \mathrm{mgR}}$ aorta to the pathophysiology of TAAD is consistent with a mechanism in which vascular SMCs increase aggrecan and versican expression, whereas their proteolysis after deposition in ECM is concomitantly reduced.

The biophysical properties of proteoglycans are key to their role in mediating aortic medial homeostasis and ECM integrity. The tissue hydration arising from a normal, physiological level of interstitial proteoglycans likely creates an optimal osmotic swelling pressure that endows the elastic lamellar unit with the ability to resist cyclic compression while simultaneously placing the elastic fibers and radially oriented microfibrilcell connections into tension $(6,9,10,22)$. The tension of ECM-cell connections may optimize mechanosensing by the vascular SMCs, leading to an appropriate adaptation of the surrounding ECM by balanced proteoglycan secretion and proteolysis. This key SMC-ECM interaction may be compromised in TAAD (14) owing to excess swelling pressure exerted by aggrecan and/or versican, which may contribute to the reported cellular maladaptation $(9,10,13)$. In addition to the biophysical impact, glycosylated versican is antiadhesive and is excluded from focal contacts (23-25). The ensuing loss of cell adhesion may also be a major factor in SMC deterioration and a maladaptive biosynthetic response. We speculate, as others have, that massive proteoglycan accumulation in TAAD is not only disruptive mechanically, but may also affect SMC viability, possibly leading to cell death by anoikis (26). Thus, medial degeneration lesions are a chasm for cells, lacking sufficient adhesive properties, promoting cell-cell and cell-ECM discontinuity, creating high stress concentrations, and resulting in a microenvironment that is less conducive to SMC survival (6). Furthermore, inclusion of aggrecan instead of, or in addition to versican in predictive models $(6,9,10)$ will likely exacerbate the previously estimated deleterious effects of proteoglycans (summarized in Figure 7).

Versican has been extensively studied in the context of vascular SMCs. Production of versican and hyaluronan is increased by transforming growth factor- $\beta$ and platelet derived growth factor, and increased intracellular and medial hyaluronan has been reported in vascular SMCs and aortic tissue, respectively, from patients with Marfan syndrome $(8,27)$. The ability of versican to inhibit elastic fiber assembly is unlikely to underlie the loss of elastic fibers in TAAD, since elastin assembly is complete by the end of the juvenile period (28). Although aggrecan has been detected in the aorta of the developing chick embryo $(28,29)$, in atherosclerotic lesions in mice deficient in the Apo E/low density lipoprotein receptor (30), in the aorto-pulmonary septum of elastin haploinsufficient mice (31), and in the ECM of the normal human aorta, it has not previously been considered as a major vascular matrix molecule. Although it was not previously investigated in TAAD (32), analysis of abdominal aortic aneurysm tissue by a quantitative proteomics approach found significantly less aggrecan than controls (33), which is intriguing since abdominal aneurysms rarely dissect (34). 
A

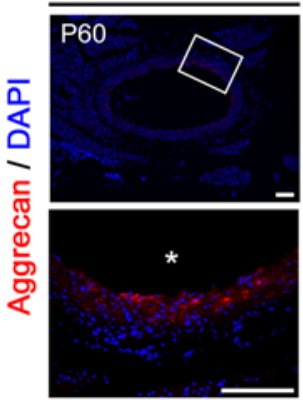

B

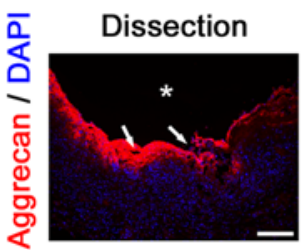

Dissected / Ruptured
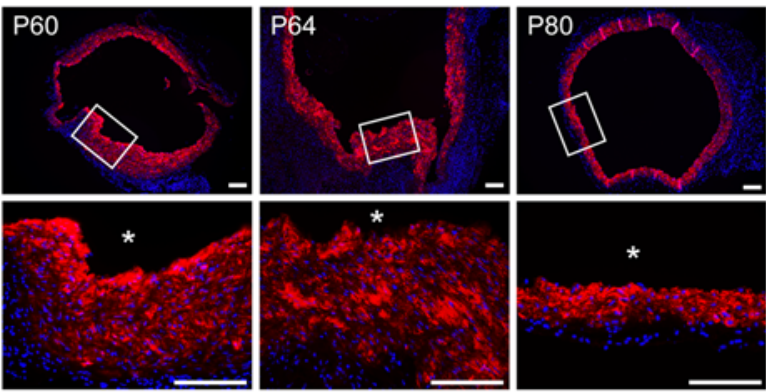

C

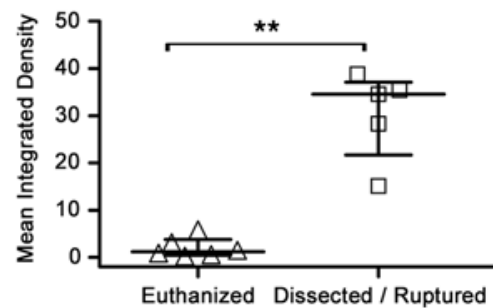

Figure 5. Severe aggrecan accumulation occurs in Fbn $1^{\mathrm{mgR} / \mathrm{mgR}}$ mice with ascending thoracic aorta dissection/rupture. (A) Aggrecan immunofluorescence in aortas of $F b n 1^{\mathrm{mgR} / \mathrm{mgR}}$ mice that were euthanized and mice that died from ascend ing thoracic aorta dissection and/or rupture. The images are representative of aortas from 6 euthanized mice and 5 dissected/ruptured thoracic aortas whose postnatal $(P)$ ages in days are indicated. The lower row contains magnified views of the boxed areas in the upper row. Scale bars: $100 \mu \mathrm{m}$; asterisks identify the aortic lumen. (B) Aggrecan is present at high levels at sites of dissection and rupture. Asterisks and arrows denote the aortic lumen and the site of dissection or rupture, respectively. These images are representative of 3 dissected/ruptured aortas where the disruption of the aortic wall was visualized histologically. (C) Quantification of aggrecan immunofluorescence in euthanized P60 and P90 Fbn $1^{\mathrm{mgR} / \mathrm{mgR}}$ mice ( $n=3$ of each, total $n=6$ ) and Fbn $1^{\mathrm{mgR} / \mathrm{mgR}}$ mice older than P45 who died with ascending aortic dissection or rupture ( $n=5$, ages P52, P60, P64, P64, and P80). Each data point represents an independent biological replicate. Differences between groups were assessed using the Mann-Whitney $U$ test with JMP Pro software (version 13). Bars represent the median with interquartile range. ${ }^{* *} P \leq 0.01$.

We therefore propose that aggrecan and/or versican accumulation in TAAD is a major contributor to dissection risk, which is supported by dramatically increased aggrecan staining seen in dissected and ruptured aortas from $F b n 1^{\mathrm{mgR} / \mathrm{mgR}}$ mice. In this mouse model, aggrecan rather than versican accumulation was noted. Since aggrecan has an order of magnitude more CS chains than versican (33), it has the potential to exert a greater swelling pressure and to be correspondingly more anti-adhesive than versican, which might explain the impact observed in the mice. $V C A N$ gene expression increased in the vascular SMCs of a patient with Loeys-Dietz Syndrome and a patient with Arterial Tortuosity Syndrome (34), as well as a cohort of patients with ascending aneurysm of unknown primary etiology (35). Our results corroborate these studies as well as a recent transcriptome analysis of aortas with acute type-A dissection that found significant downregulation of ADAMTS5 relative to control (36). Overall, the findings suggest a scenario where TAAD SMCs, surrounded by excess proteoglycans, do turn them over proteolytically, but not to a degree that prevents their accumulation over time before succumbing to cell death via anoikis. In this event, the proteoglycan pool persists, serving as the tombstone of the SMC. The data show that proteoglycan pooling occurs at multiple sites within the aorta, presumably over extended time scales in humans with a TAAD-causing primary pathology, progressively weakening the structure until vessel-level failure occurs in the form of an aortic dissection. In $F b n 1^{\mathrm{mgR} / \mathrm{mgR}}$ mice, which present a genetically homogeneous model of severe Marfan syndrome, the observed aggrecan accumulation showed a significant association with dissection and rupture. We have not yet undertaken a similar analysis in human TAAD, because of the broad spectrum of underlying causes, requiring analysis of a large number of cases representing each primary etiology. This is an important and potentially challenging area for future research. We speculate that proteoglycan accumulation may not only occur in TAAD as a formal disease process, but as a chronic degenerative phenomenon contributing to aging of the aorta (5). We further speculate that this phenomenon is accelerated in TAAD by underlying genetic defects in the cell-ECM continuum. 
A

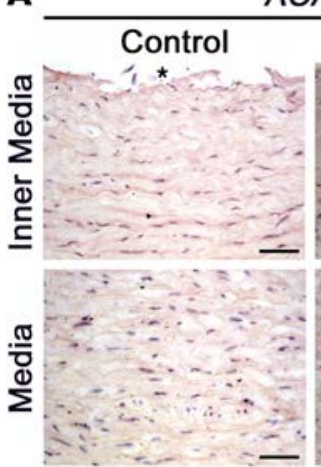

ACAN
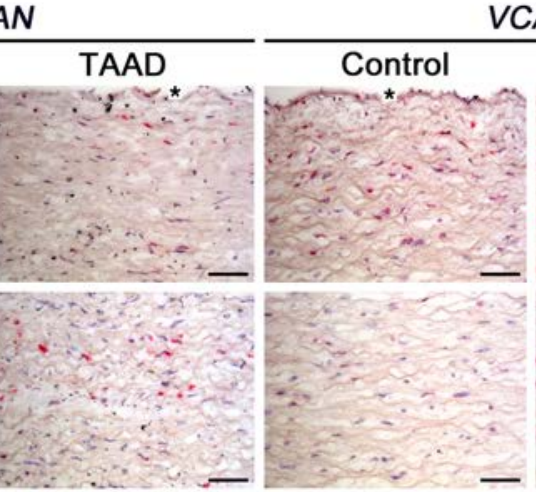

VCAN

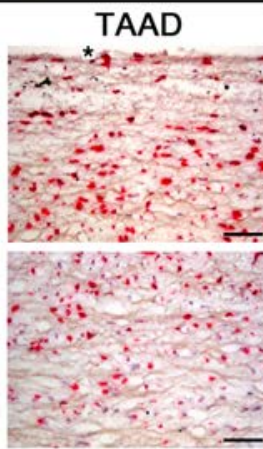

B

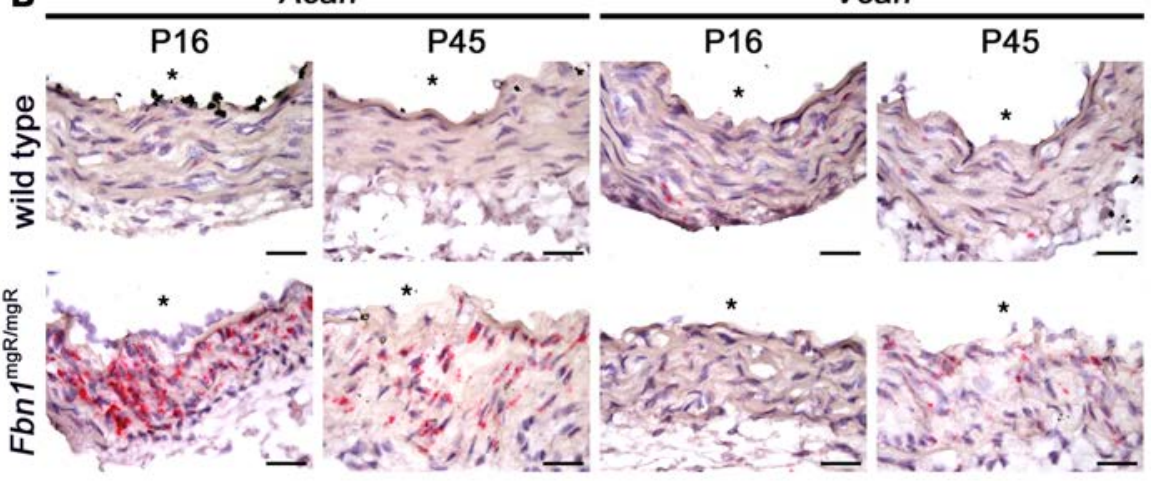

Figure 6. RNA in situ hybridization (ISH) shows increased aggrecan and versican gene expression in human and mouse ascending thoracic aortic aneurysm and dissection (TAAD). (A) ISH revealed little $A C A N$ expression in control aorta. In human TAAD, clusters of cells with high ACAN expression (red signal) were seen in areas of disorganized elastic fibers. VCAN ISH demonstrated dramatically increased expression in TAAD samples relative to control. The asterisks indicate the luminal aspect. The images are representative of ISH of 3 control and 3 TAAD cases. Scale bars: $50 \mu \mathrm{m}$. (B) Acan and Vcan mRNA expression is increased in $\mathrm{Fbn} 1^{\mathrm{mgR} / \mathrm{mgR}}$ aortas versus wild type at P16 and P45, with more dramatic upregulation of Acan than Vcan. The images are representative of 4 wild-type and $4 \mathrm{Fbn} 7^{\mathrm{mgR} / \mathrm{mgR}}$ aortas at each time point. Scale bars: $20 \mu \mathrm{m}$.

Although proteoglycan pooling has long been recognized as a histopathologic hallmark of TAAD, it has only recently been implicated in the pathogenesis of dissection $(6,9,10,37)$. A previous immunohistochemical characterization of proteoglycans in aortic dissection concluded that versican was neither present in areas of medial degeneration nor increased in patients with aortic dissection (38). The use of mass spectrometry together with immunofluorescence using well-characterized aggrecan and versican antibodies in the present study provides unequivocal evidence of their presence in normal aorta and TAAD, especially in medial degeneration lesions. Another study identified increased CS in TAAD tissue compared with controls, supporting the present findings (39)

Analysis of the $F b n 1^{\mathrm{mgR} / \mathrm{mgR}}$ mice provided several potentially novel insights. The temporal pattern of aggrecan accumulation in the $F b n I^{\mathrm{mgR} / \mathrm{mgR}}$ mouse is consistent with the likelihood of aggrecan being responsible, at least in part, for aortic structural vulnerability and initiating medial delamination, as half of these mutant mice typically succumb to aortic dissection/rupture at 2.5 months (18). The finding that wild-type aortas contained ample cleaved aggrecan while $F b n 1^{\mathrm{mgR} / \mathrm{mgR}}$ aortas did not, suggests that aggrecan accumulation may arise from both upregulation of Acan mRNA and reduced proteolytic clearance.

Overall, the findings show that aortic dissection/rupture is consistently associated with accumulation of aggrecan in the tunica media (in the $F b n 1^{\mathrm{mgR} / \mathrm{mgR}}$ mice), suggesting that aggrecan may be an important contributor to dissection pathogenesis and may serve as a biomarker of aortic disease progression/ dissection risk in humans, which requires further investigation. Unexpectedly, nearly all aortas of both wild-type and $F b n 1^{\mathrm{mgR} / \mathrm{mgR}}$ mice were devoid of versican at P16, P30, P45, P60, and P90, and wild-type aorta was nearly devoid of any immunoreactive aggrecan and versican at P30, P45, P60, and P90. This finding suggests that the postnatal murine aorta normally has a low steady-state versican content and both versican and aggrecan may be turned over rapidly, potentially by ADAMTS proteases. Although relatively little is known about the specific roles of ADAMTS proteases in vascular SMCs and the aorta,

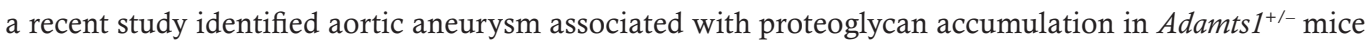
(40). Since mouse models are widely used for understanding human TAAD, the disparity in versican accumulation in TAAD and $F b n 1^{\mathrm{mgR} / \mathrm{mgR}}$ aortas is a noteworthy difference.

In conclusion, this work suggests that accumulation of large aggregating proteoglycans arising from the combination of increased production and reduced proteolysis may be a consistent and deleterious event in 

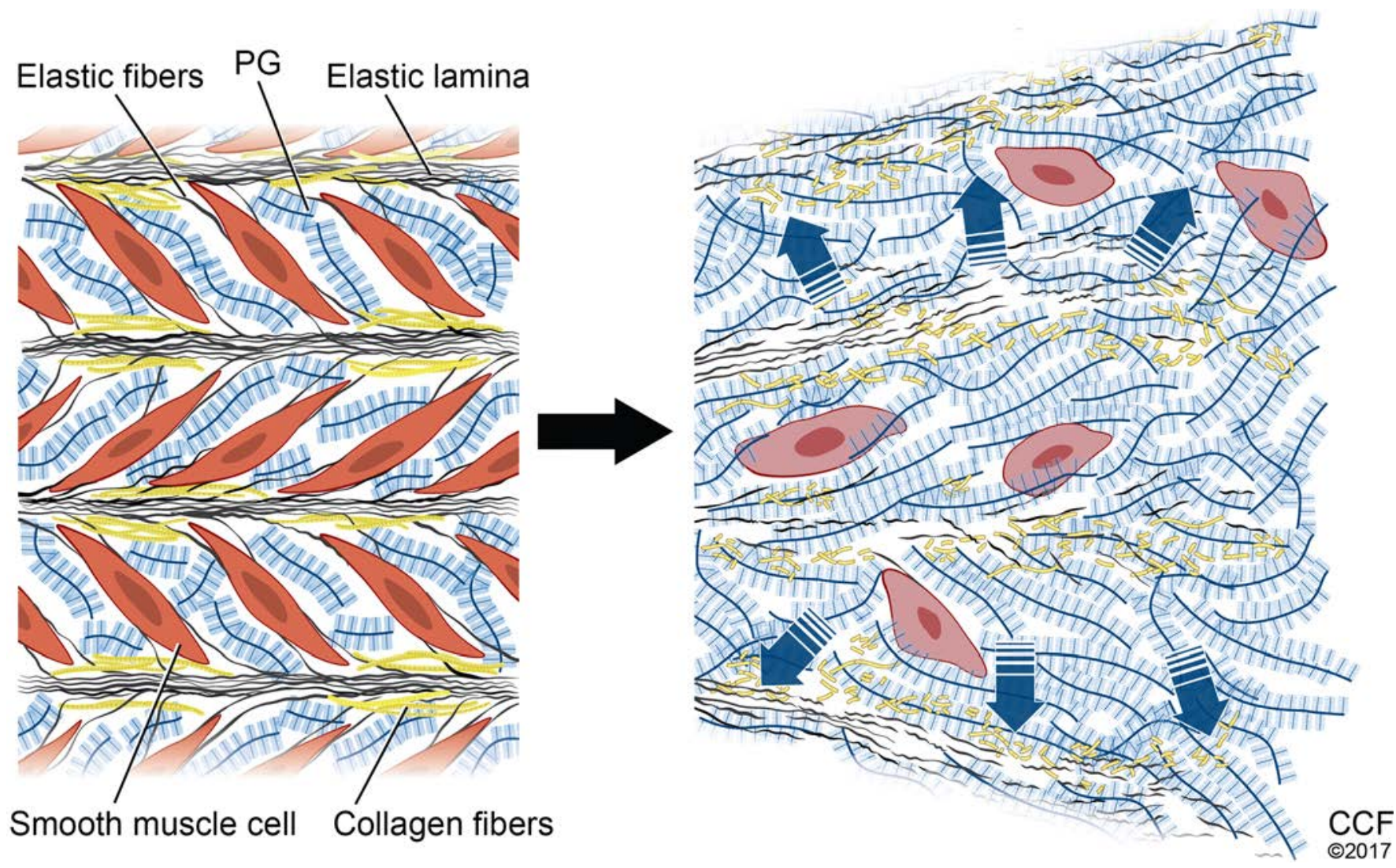

Figure 7. Model depicting the impact of large aggregating proteoglycan accumulation in ascending thoracic aortic aneurysm and dissection (TAAD). In the normal aorta (left) the proteoglycans (PGs) aggrecan and versican as well as glycosaminoglycans provide compressive stiffness to the elastic lamellar units and place elastic fiber/microfibril-cell connections into tension. Pathologic accumulation of proteoglycans in TAAD (right), together with elastic fiber fragmentation may reduce smooth muscle cell-extracellular matrix connections. This loss of adhesive and mechanical input is proposed to perturb vascular smooth muscle cell mechanosensing and viability, depicted here as loss of the normal smooth muscle cell spindle shape and rounding in TAAD. Illustration is by David Schumick and reprinted with the permission of the Cleveland Clinic Center for Medical Art \& Photography @ 2017. All rights reserved.

TAAD regardless of primary etiology. The findings strengthen the evidence for a disruptive role for proteoglycan accumulation in TAAD biomechanics, and identify aggrecan and versican as potential biomarkers for disease severity and risk of aortic dissection. Additionally, further understanding the regulation of proteoglycan dynamics may provide new targets for nonsurgical therapy of TAAD.

\section{Methods}

Human aorta specimens. Cases of ascending TAAD undergoing elective surgery (ascending aneurysm) or emergency surgical repair (acute type-A dissection) were prospectively enrolled in an approved study after written informed consent was obtained from participants or family members. Participants with ascending TAAD due to any primary etiology were included, although suspected autoimmune aortitis, chronic aortic dissection, or iatrogenic dissection were excluded. Maximum ascending aortic diameter was collected from contrast-enhanced computed tomography scans in most participants, and transesophageal echocardiography was used in 2 participants. The presence of aortic valve disease was assessed with preoperative echocardiography and bicuspid aortic valve was confirmed via direct inspection in the operating room. The diagnosis of connective tissue disease was made via clinical genetic testing when desired by the participants and obtained from the medical records. Clinical details are listed in Supplemental Table 1. Ascending aortas collected from heart transplant donor tissue provided by Lifebanc that were not utilized for transplantation for reasons other than the health of the tissue, or patients undergoing aortic valve surgery without aneurysmal disease were used as controls. Tissue was frozen immediately after resection and stored at $-80^{\circ} \mathrm{C}$. Resected aortas were collected prospectively from 36 participants with ascending aortic aneurysms and 21 participants with acute type-A dissections. The patients encompassed a representative cross section 
of known causes of TAAD, with the majority of cases being idiopathic (Table 2). Aortas from 5 organ donors and 3 participants undergoing valve replacement served as controls.

Mouse model of severe Marfan syndrome. Mice with targeted mutagenesis of the Fbn1 locus that reduces the level of fibrillin-1 to approximately $20 \%$ of normal were previously described (14). The mice were backcrossed to $100 \%$ purity (>10 generations) in the C57BL/6J strain in 1996 and subsequently maintained by biannual backcrosses to this strain. Ascending aortas were obtained after euthanasia from homozygous mutant males $\left(F b n^{\mathrm{mgR} / \mathrm{mgR}}\right.$ ) and wild-type littermate male mice at P16, P30, P45, P60, and P90 ( $n \geq 3$ for each group) or after natural, but premature death due to aortic dissection and/or rupture. Necropsy was performed on these dead mice and they were determined to have died of aortic dissection/rupture if a substantial amount of blood was found on entering the thoracic cavity. The aorta was processed and tissue was fixed in 10\% neutral-buffered formalin overnight prior to paraffin embedding.

Proteoglycan isolation. Aorta proteoglycans were isolated and analyzed from 3 ascending thoracic aortic aneurysms and 3 control ascending aortas according to the schematic shown in Figure 1A. Aorta samples with intact intimal, medial, and adventitial layers but having supra-adventitial connective tissue and fat removed were partially thawed. Tissue $(600 \mathrm{mg}$ ) was placed in $6 \mathrm{ml}$ proteoglycan extraction buffer (4 M guanidine hydrochloride, 2\% 3-[(3-cholamidopropyl) dimethylammonio]-1-propanesulfonate [CHAPS], 50 $\mathrm{mM}$ sodium acetate, $\mathrm{pH}$ 6.0) containing 1 tablet of cOmplete Mini EDTA-free Protease Inhibitor (Roche). The tissue was homogenized using an Ultra-Turrax T2 or T10 homogenizer (IKA Works Inc.) and rotated end-over-end for approximately 16 hours at $4^{\circ} \mathrm{C}$. The homogenate was centrifuged for 15 minutes at $20,000 \mathrm{~g}$ and the supernatant (aorta protein extract) was retained. Sephadex G50 Fine (Amersham Biosciences) sizeexclusion chromatography was utilized to exchange the proteoglycan extraction buffer into G50 buffer ( 8 M urea, $0.5 \%$ CHAPS, $50 \mathrm{mM}$ sodium acetate, $150 \mathrm{mM} \mathrm{NaCl}, \mathrm{pH}$ 7.0) and render the extract compatible with anion-exchange chromatography. The aorta protein extract was loaded onto a 4-ml diethylaminoethyl (DEAE)-Sephacel column (Sigma-Aldrich) and washed with 5 column volumes of G50 buffer containing $250 \mathrm{mM} \mathrm{NaCl}$. Strongly anionic proteins were eluted in a single step with G50 buffer containing $1 \mathrm{M} \mathrm{NaCl}$. Proteoglycan-containing fractions were identified by safranin-O dot blot by extracting precipitated safranin-O from the nitrocellulose membrane with $10 \%$ cetylpyridinium chloride and measuring absorbance at $560 \mathrm{~nm}$ (Cytation 5, BioTek Instruments) (41). Additionally, protein concentration was determined by UV absorbance at $280 \mathrm{~nm}$ (Nanodrop 1000, Thermo Fisher Scientific). Proteoglycan-containing fractions were pooled and dialyzed into $20 \mathrm{mM}$ (4-[2-hydroxyethyl]-1-piperazineethanesulfonic acid) (HEPES) with $150 \mathrm{mM} \mathrm{NaCl}$.

FACE. For validation of the isolation protocol, DEAE elution fractions were analyzed by FACE for GAG content as previously described $(42,43)$. Briefly, proteins were digested with proteinase $\mathrm{K}$ and removed by ammonium acetate/methanol precipitation. The remaining glycans were digested with $0.02 \mathrm{U} /$ sample Proteus vulgaris chondroitinase ABC (C2905-10UN, Sigma-Aldrich), 0.125 U/sample Streptococcus dysgalactiae hyaluronidase (100741-1A, Seikagaku), $0.02 \mathrm{U} /$ sample Flavobacterium heparinum heparinase I and III blend, and 5 U/sample heparinase II (H3917-50UN and H6512-10UN, respectively; Sigma-Aldrich). The remaining saccharides and disaccharides were then fluorescently labeled with 2-aminoacridone and analyzed by polyacrylamide gel electrophoresis. The gel was imaged using a ChemiDoc-It2 515 integrated imaging system (UVP, LLC).

Western blotting. Samples were digested with $0.001 \mathrm{U} / \mu 1$ chondroitinase ABC from $P$. vulgaris (3667, Sigma-Aldrich) for 2 hours at $37^{\circ} \mathrm{C}$, reduced using $5 \%$ (v/v) $\beta$-mercaptoethanol, and denatured by boiling at $100^{\circ} \mathrm{C}$ for 5 minutes prior to loading onto a $7.5 \%$ SDS polyacrylamide gel. Gels were electroblotted to a polyvinyldifluoridene (PVDF) membrane (Immobilon - FL, EMD Millipore Corp.) for 1 hour at 333 $\mathrm{mA}$ in Tris $(25 \mathrm{mM})$ - glycine $(192 \mathrm{mM})$ buffer containing $20 \%$ methanol. The membrane was blocked for 1 hour at room temperature with gentle rocking using Odyssey Blocking Buffer (927-50000, Li-Cor). Primary antibody was diluted in Odyssey Blocking Buffer and incubated with gentle rocking for 16 hours at $4^{\circ} \mathrm{C}$. The membrane was washed 3 times for 5 minutes each wash with gentle rocking using TBST (10 mM Tris-HCl, 150 mM NaCl, pH 7.2, 0.1\% Tween 20). Fluorophore-labeled IRDye goat anti-mouse or goat anti-rabbit secondary antibodies were diluted 1:10,000 in Odyssey Blocking Buffer and incubated with the membrane for 1 hour at room temperature with gentle rocking in the dark. The membrane was washed 3 times for 5 minutes each wash with gentle rocking using TBST followed by one 5 -minute wash with gentle rocking in TBS (10 mM Tris- $\mathrm{HCl}, 150 \mathrm{mM} \mathrm{NaCl}, \mathrm{pH}$ 7.2) in the dark. The protein-bound antibodies were visualized on the membrane using infrared fluorescence detection for Western blotting (Li-Core Biosciences Odyssey CLx imaging system). 
Table 2. Participant demographics and clinical data

\begin{tabular}{|c|c|c|c|}
\hline & $\begin{array}{l}\text { Control } \\
(n=8)\end{array}$ & $\begin{array}{l}\text { Thoracic ascending aneurysm } \\
\qquad(n=36)\end{array}$ & $\begin{array}{l}\text { Acute type-A dissection } \\
\qquad(n=21)\end{array}$ \\
\hline Age (years) & $60[12.2]$ & $62[17.7]$ & $62[20.5]$ \\
\hline Gender (\% male) & 50.0 & 72.2 & 57.1 \\
\hline TAAD etiology $(n)$ & $N / A$ & & \\
\hline Idiopathic & & 13 & 20 \\
\hline BAV & & 17 & 1 \\
\hline CTD & & 6 & 0 \\
\hline Aortic Diameter (mm) & $\mathrm{N} / \mathrm{A}$ & $50[11]$ & $46[8]$ \\
\hline Comorbidities (\%) & $N / A$ & & \\
\hline Hypertension & & 58.0 & 81.0 \\
\hline Coronary artery disease & & 22.2 & 14.3 \\
\hline Stroke & & 5.6 & 14.3 \\
\hline Chronic kidney disease & & 5.6 & 4.8 \\
\hline Type II diabetes & & 8.3 & 9.5 \\
\hline Current smoker & & 8.3 & 9.5 \\
\hline \multicolumn{4}{|c|}{$\begin{array}{l}\text { Continuous variables are expressed as median [IQR]. BAV, bicuspid aortic valve; CTD, connective tissue disease, } \\
\text { including Marfan syndrome }(n=2) \text {, Loeys-Dietz syndrome }(n=1) \text {, vascular Ehlers-Danlos syndrome }(n=2) \text {, and familial } \\
\text { thoracic aortic aneurysm and dissection }(n=1) \text {. Please see Supplemental Table } 1 \text { for additional participant details. Aorti } \\
\text { diameter and comorbidity data were not available (N/A) for controls. }\end{array}$} \\
\hline
\end{tabular}

Proteomic analysis of isolated proteoglycans. Aorta proteoglycans equivalent to $20 \mu \mathrm{g}$ total protein were lyophilized in a SpeedVac evaporator, reconstituted in $50 \mu 16 \mathrm{M}$ urea, $100 \mathrm{mM}$ Tris, $\mathrm{pH}$ 7.0, reduced by addition of $2.5 \mu 1200 \mathrm{mM}$ dithiothreitol and alkylated using $200 \mathrm{mM}$ iodoacetamide. The urea concentration was reduced to $1.2 \mathrm{M}$ by diluting the sample with $160 \mu \mathrm{l}$ double-distilled water and the $\mathrm{pH}$ was adjusted to $>8.0$ with $100 \mathrm{mM}$ ammonium bicarbonate. Samples underwent consecutive overnight digests using trypsin at an enzyme/protein ratio of 1:20 at room temperature. The tryptic peptide mixture was desalted using Pierce C18 spin columns (Thermo Fisher Scientific), lyophilized in a SpeedVac evaporator, reconstituted in $30 \mu 1$ \% acetic acid, and separated on a Dionex Ultimate 3000 nanoflow UHPLC system using a $75 \mu \mathrm{m} \times 15 \mathrm{~cm}, 3-\mu \mathrm{m}$ particle size, Acclaim PepMap $100 \mathrm{C} 18$ column (Thermo Fisher Scientific) at a flow rate of $0.3 \mu 1 /$ minute. Peptides were eluted using buffers A $(0.1 \%$ formic acid in water $)$ and $\mathrm{B}(0.1 \%$ formic acid in acetonitrile) with a stepwise gradient. After sample loading over 5 minutes with $2 \%$ buffer $B$, elution was performed with a continuous gradient of $2 \%-40 \%$ buffer B from 5-110 minutes, followed by a continuous gradient of $40 \%-80 \%$ buffer B over 5 minutes, and finally with $80 \%$ buffer B for an additional 5 minutes. The UHPLC column was coupled to a nanospray source through a PicoTip emitter (FS360-2015-N-20-C15, New Objective). An LTQ-Orbitrap-Elite high-resolution hybrid mass spectrometer (Thermo Fisher Scientific) was used to collect spectra using a full-ion scan at a resolution of 60,000 over the mass/ charge range 300-2,000. MS $^{2}$ scans using collision-induced dissociation were performed on the 20 most abundant precursor ions from $\mathrm{MS}^{1}$ scans using the data-dependent mode with dynamic exclusion. $\mathrm{MS}^{2}$ spectra were matched to the UniProtKB/Swiss-Prot human database using Proteome Discoverer. The percolator function was utilized to select only matches with a $Q$ value $<0.01(<1 \%$ false discovery rate [FDR]). The mass tolerance was $10 \mathrm{ppm}$ for precursor ions and $0.8 \mathrm{Da}$ for $\mathrm{MS}^{2}$. Carboxyamidomethylation of cysteine was set as a fixed modification and oxidation of methionine was set as a variable modification. Only full tryptic peptides were considered with a maximum of 3 missed cleavage sites.

Histology and immunofluorescence. Full-thickness tissue blocks from the anterolateral aspect of human ascending aortas were fixed in $4 \%$ paraformaldehyde for 48 hours at $4^{\circ} \mathrm{C}$. The samples were paraffin embedded for sectioning in the circumferential orientation and $6-\mu \mathrm{m}$-thick sections were stained with Movat pentachrome, Alcian blue, and Masson trichrome stains. Serial sections with areas of medial degeneration were selected for an indirect immunofluorescence method from 20 TAAD cases. Six control aortas were utilized. Sections were heated in citrate buffer, $\mathrm{pH} 6.0$ for antigen retrieval and allowed to cool to room temperature, treated with chondroitinase $\mathrm{ABC}(0.05 \mathrm{U} / \mu 1$, diluted 1:200 in PBST $(137 \mathrm{mM} \mathrm{NaCl}, 2.7 \mathrm{mM} \mathrm{KCl}, 4.3 \mathrm{mM}$ $\mathrm{Na}_{2} \mathrm{HPO}_{4}, 10 \mathrm{mM}$ Tris- $\mathrm{HCl}, 150 \mathrm{mM} \mathrm{NaCl}, 0.1 \%$ Tween $20, \mathrm{pH}$ 7.2) with $5 \%$ normal goat serum), blocked 
for 1 hour at room temperature, and stained with antibodies against versican (anti-VC), which detects intact and cleaved versican (cleavage at the $\mathrm{E}^{441}-\mathrm{A}^{442}$ peptide bond; V1 isoform enumeration) (44), and aggrecan (polyclonal AB1031, and mouse monoclonal MCA1454G). Rabbit polyclonal antibodies against an ADAMTS protease-generated aggrecan neoepitope $\left(\mathrm{NITEGE}^{393}\right.$ ) and versican neoepitope (DPEAAE ${ }^{441}$; V1 enumeration) were used to localize aggrecan and versican cleavage, respectively. Complete details of antibodies are listed in Supplemental Table 2. Alexa Fluor secondary goat anti-mouse or goat anti-rabbit antibodies (Invitrogen) were diluted 1:200 in PBST and incubated on the tissue sections for 1 hour at room temperature protected from light. Slides were washed 3 times for 5 minutes each wash using PBST and 1 time for 5 minutes using PBS $\left(137 \mathrm{mM} \mathrm{NaCl}, 2.7 \mathrm{mM} \mathrm{KCl}, 4.3 \mathrm{mM} \mathrm{Na} \mathrm{HPO}_{4}, 10 \mathrm{mM}\right.$ Tris-HCl, 150 $\mathrm{mM} \mathrm{NaCl}$ ) in the dark, mounted using Prolong-Gold with the DNA label 4',6-diamidino-2-phenylindole (DAPI, Thermo Fisher Scientific) and imaged on an Olympus BX51 microscope with a Leica DFC7000 T camera using Leica Application Suite software. ImageJ (NIH) or Adobe Photoshop software was used for postprocessing of images, specifically, white/black balancing and adjustment of brightness and contrast as needed for clarity, with the adjustments being applied equally to the entire image for all images.

Immunofluorescence within the intima and media was quantified with ImageJ software using the region of interest (ROI) tool when the aortic tissue did not occupy the entire field of view. Mean integrated density was measured for the ROIs. All quantification was performed on images taken with the same exposure settings and without postimage processing, with the exception of color thresholding applied equally to all images to reduce background signal. The individual data points along with the median and IQR for each group was plotted using GraphPad Prism (version 5).

$R N A$ analyses. Acan and Vcan mRNA in situ hybridization was performed using RNAScope (Advanced Cell Diagnostics) following the manufacturer's protocol. Briefly, 7- $\mu \mathrm{m}$ sections were deparaffinized and hybridized to human and mouse aggrecan and versican probe sets (ACAN, 506841; VCAN, 452241; Acan, 506841; Vcan, 428311, Advanced Cell Diagnostics) using a HybEZ oven (Advanced Cell Diagnostics) and the RNAScope 2.5 HD Detection Reagent Kit (322360, Advanced Cell Diagnostics).

For RT-qPCR, frozen human aortas were partially thawed and approximately 100-mg full-thickness fragments from the proximal, antero-lateral aspect of the ascending aorta were obtained. Tissue was minced, total RNA was isolated using TRIzol (Invitrogen), and $1 \mu \mathrm{g}$ of RNA was reverse transcribed into cDNA (SuperScript III Cellsdirect cDNA synthesis system, 46-6321, Invitrogen). RT-qPCR was performed with Bullseye EvaGreen qPCR MasterMix (BEQPCR-S, MIDSCI) using a CFX96 thermal cycler (BioRad Laboratories) for the following genes: ACAN, VCAN, ADAMTS1, ADAMTS4, ADAMTS5, ADAMTS9, $A D A M T S 15$, and ADAMTS20 (primer sequences available in Supplemental Table 3). GAPDH was used to normalize mRNA quantity.

RNA-sequencing (RNA-seq) was carried out on total RNA purified from the aortas of P16, P30, and P60 Fbn $1^{\mathrm{mgR} / \mathrm{mgR}}$ mice ( $n=3$ per group). Aortas were flash-frozen and total RNA was extracted using the RNeasy Fibrous Tissue Mini Kit (Qiagen) with DNase treatment. Samples with an RNA integrity score above 8 were used to make a poly(A)-primed cDNA library that was sequenced using the Illumina HiSeq 4000 system. Sequencing reads were aligned to the mouse reference genome 'mm9' using Tophat 2.0.8, samtools-0.1.7, and bowtie 2.1.0. Differentially expressed genes (DEGs) were identified with Cufflinks 1.3.0. Tophat was used with the ensemble GTF file as a gene annotation reference and the option 'nonovel-juncs.' Output BAM files were directly subjected to differential gene expression identification using Cufflinks with the options 'multi-read-correct,' 'upper-quartile-norm,' and 'frag-bias-correct' against the mm9 genome. DEGs in mutant versus wild-type aortas were identified based on an FDR of $10 \%$ and a minimum fold change calculated using $\log 2\left(\left[\mathrm{FPKM}_{\text {condition } 1}+1\right] /\left[\mathrm{FPKM}_{\text {condition2 }}+1\right]\right) \geq \pm \log 2(1.3)$, where FPKM is fragments per kilobase of transcript per million mapped reads. Values for genes of interest were extracted from the RNA-seq data for the present study. The data are available in the NCBI's Gene Expression Omnibus (GEO GSE109722).

Statistics. Immunofluorescence experiments were performed with at least 3 independent biological replicates for each group. The median and IQR was calculated for each replicate using GraphPad Prism (version 5). This is expressed in the text as median [IQR]. Differences between groups were assessed using the Mann-Whitney $U$ test with JMP Pro software (version 13, SAS). $P$ values $\leq 0.05$ were considered statistically significant.

RT-qPCR experiments were performed with 3 independent samples and confirmed reproducibility. The $\Delta \Delta \mathrm{Ct}$ method was used to calculate relative mRNA expression levels of target genes. A 2-tailed Student's $t$ test with significance level $(P)$ of $<0.05$ was used. 
Study approval. Prospective collection of aortic aneurysm and dissection tissue and of donor control tissue was approved by the Cleveland Clinic Institutional Review Board (IRB 16-900 and IRB 2378). Mice were used under a protocol (no. 08-276) approved by the Institutional Animal Care and Use Committee of the Icahn School of Medicine at Mt. Sinai.

\section{Author contributions}

FSC, CDK, TJM, JG, BBW, FR, and SSA designed the experiments. FSC, CDK, TJM, and JG performed experiments and analyzed the data. $\mathrm{KBE}$ initiated the project and discussed the findings. MJE and EHB discussed the findings. EER provided patient material and discussed the findings. FSC, CDK, and SSA wrote the manuscript. FSC and CDK made the figures. All authors edited the manuscript and approved it for submission.

\section{Acknowledgments}

We thank TAAD participants, organ donors, and their families for consenting to the use of tissue in the present report. This work was supported by the NIH-NHLBI Program of Excellence in Glycosciences (HL107147 to S.S. Apte), American Heart Association Paul G. Allen Frontiers Group Distinguished Investigator Award (to S.S. Apte), Sabrina's Foundation (to C.D. Koch and S.S. Apte.), NIH-NIAMS (award AR069307 to F. Ramirez), a grant from W.L. Gore, Inc. (F.S. Cikach, K.B. Emerton, M.J. Eagleton, E.E. Roselli), the High Risk Cardiovascular Research Fund (to E.E. Roselli), and a grant from the Cleveland Clinic Research Program Committees (RPC grant 202 to F.S. Cikach). We thank Katrina Owens for paraffin sectioning and histochemical stains, Ling Li and Dongmei Zhang for proteomic support, the NIH-supported Program of Excellence in Glycosciences-supported Glycobiology Core, and Valbona Cali for FACE analysis, members of the Apte laboratory for helpful discussion and cardiovascular surgeons and surgical staff of the Cleveland Clinic for tissue procurement. The differentially expressed ECM genes shown in Supplemental Table 4 were extracted from unpublished analyses of RNA-Seq data by Jens Hansen and Ravi Iyengar. Research reported in this publication was supported by the Office of the Director, NIH under award number S10RR031537 for purchase of the mass spectrometer. The content is solely the responsibility of the authors and does not necessarily represent the official views of the NIH.

Address correspondence to: Suneel S. Apte, Department of Biomedical Engineering-ND20, Cleveland Clinic Lerner Research Institute, 9500 Euclid Avenue, Cleveland, Ohio 44195, USA. Phone: 216.445.3278; Email: aptes@ccf.org.

MJE's present address is: Massachusetts General Hospital Division of Vascular and Endovascular Surgery, Boston, Massachusetts, USA.

1. Humphrey JD, Milewicz DM, Tellides G, Schwartz MA. Cell biology. Dysfunctional mechanosensing in aneurysms. Science 2014;344(6183):477-479.

2. Humphrey JD, Schwartz MA, Tellides G, Milewicz DM. Role of mechanotransduction in vascular biology: focus on thoracic aortic aneurysms and dissections. Circ Res. 2015;116(8):1448-1461.

3. Lindsay ME, Dietz HC. Lessons on the pathogenesis of aneurysm from heritable conditions. Nature. 2011;473(7347):308-316.

4. Milewicz DM, et al. Altered smooth muscle cell force generation as a driver of thoracic aortic aneurysms and dissections. Arterioscler Thromb Vasc Biol. 2017;37(1):26-34.

5. Halushka MK, et al. Consensus statement on surgical pathology of the aorta from the Society for Cardiovascular Pathology and the Association For European Cardiovascular Pathology: II. Noninflammatory degenerative diseases - nomenclature and diagnostic criteria. Cardiovasc Pathol. 2016;25(3):247-257.

6. Humphrey JD. Possible mechanical roles of glycosaminoglycans in thoracic aortic dissection and associations with dysregulated transforming growth factor- $\beta$. J Vasc Res. 2013;50(1):1-10.

7. Azeloglu EU, Albro MB, Thimmappa VA, Ateshian GA, Costa KD. Heterogeneous transmural proteoglycan distribution provides a mechanism for regulating residual stresses in the aorta. Am J Physiol Heart Circ Physiol. 2008;294(3):H1197-H1205.

8. Evanko SP, Angello JC, Wight TN. Formation of hyaluronan- and versican-rich pericellular matrix is required for proliferation and migration of vascular smooth muscle cells. Arterioscler Thromb Vasc Biol. 1999;19(4):1004-1013.

9. Roccabianca S, Ateshian GA, Humphrey JD. Biomechanical roles of medial pooling of glycosaminoglycans in thoracic aortic dissection. Biomech Model Mechanobiol. 2014;13(1):13-25.

10. Roccabianca S, Bellini C, Humphrey JD. Computational modelling suggests good, bad and ugly roles of glycosaminoglycans in arterial wall mechanics and mechanobiology. JR Soc Interface. 2014;11(97):20140397.

11. Wight TN, Merrilees MJ. Proteoglycans in atherosclerosis and restenosis: key roles for versican. Circ Res. 2004;94(9):1158-1167.

12. Ateshian GA, Rajan V, Chahine NO, Canal CE, Hung CT. Modeling the matrix of articular cartilage using a continuous fiber 
angular distribution predicts many observed phenomena. J Biomech Eng. 2009;131(6):061003.

13. Chandran PL, Horkay F. Aggrecan, an unusual polyelectrolyte: review of solution behavior and physiological implications. Acta Biomater. 2012;8(1):3-12.

14. Bunton TE, Biery NJ, Myers L, Gayraud B, Ramirez F, Dietz HC. Phenotypic alteration of vascular smooth muscle cells precedes elastolysis in a mouse model of Marfan syndrome. Circ Res. 2001;88(1):37-43.

15. Evanko SP, Johnson PY, Braun KR, Underhill CB, Dudhia J, Wight TN. Platelet-derived growth factor stimulates the formation of versican-hyaluronan aggregates and pericellular matrix expansion in arterial smooth muscle cells. Arch Biochem Biophys. 2001;394(1):29-38.

16. Didangelos A, Yin X, Mandal K, Baumert M, Jahangiri M, Mayr M. Proteomics characterization of extracellular space components in the human aorta. Mol Cell Proteomics. 2010;9(9):2048-2062.

17. Pereira L, et al. Pathogenetic sequence for aneurysm revealed in mice underexpressing fibrillin-1. Proc Natl Acad Sci USA 1999;96(7):3819-3823

18. Cook JR, et al. Dimorphic effects of transforming growth factor- $\beta$ signaling during aortic aneurysm progression in mice suggest a combinatorial therapy for Marfan syndrome. Arterioscler Thromb Vasc Biol. 2015;35(4):911-917.

19. Dupuis LE, et al. Altered versican cleavage in ADAMTS5 deficient mice; a novel etiology of myxomatous valve disease. Dev Biol. 2011;357(1):152-164.

20. Fosang AJ, Rogerson FM. Identifying the human aggrecanase. Osteoarthr Cartil. 2010;18(9):1109-1116.

21. Nandadasa S, Foulcer S, Apte SS. The multiple, complex roles of versican and its proteolytic turnover by ADAMTS proteases during embryogenesis. Matrix Biol. 2014;35:34-41.

22. Davis EC. Smooth muscle cell to elastic lamina connections in developing mouse aorta. Role in aortic medial organization. Lab Invest. 1993;68(1):89-99.

23. Lemire JM, Merrilees MJ, Braun KR, Wight TN. Overexpression of the V3 variant of versican alters arterial smooth muscle cell adhesion, migration, and proliferation in vitro. J Cell Physiol. 2002;190(1):38-45.

24. Yamagata M, Kimata K. Repression of a malignant cell-substratum adhesion phenotype by inhibiting the production of the anti-adhesive proteoglycan, PG-M/versican. J Cell Sci. 1994;107(Pt 9):2581-2590.

25. Yamagata M, Saga S, Kato M, Bernfield M, Kimata K. Selective distributions of proteoglycans and their ligands in pericellular matrix of cultured fibroblasts. Implications for their roles in cell-substratum adhesion. J Cell Sci. 1993;106(Pt 1):55-65.

26. Michel JB. Anoikis in the cardiovascular system: known and unknown extracellular mediators. Arterioscler Thromb Vasc Biol. 2003;23(12):2146-2154

27. Nataatmadja M, West J, West M. Overexpression of transforming growth factor-beta is associated with increased hyaluronan content and impairment of repair in Marfan syndrome aortic aneurysm. Circulation. 2006;114(1 Suppl):I371-I377.

28. Wagenseil JE, Mecham RP. Vascular extracellular matrix and arterial mechanics. Physiol Rev. 2009;89(3):957-989.

29. Arciniegas E, Neves CY, Candelle D, Parada D. Differential versican isoforms and aggrecan expression in the chicken embryo aorta. Anat Rec A Discov Mol Cell Evol Biol. 2004;279(1):592-600.

30. Ström A, Ahlqvist E, Franzén A, Heinegård D, Hultgårdh-Nilsson A. Extracellular matrix components in atherosclerotic arteries of Apo E/LDL receptor deficient mice: an immunohistochemical study. Histol Histopathol. 2004;19(2):337-347.

31. Krishnamurthy VK, et al. Asymmetric cell-matrix and biomechanical abnormalities in elastin insufficiency induced aortopathy. Ann Biomed Eng. 2014;42(10):2014-2028.

32. Schönherr E, Järveläinen HT, Sandell LJ, Wight TN. Effects of platelet-derived growth factor and transforming growth factorbeta 1 on the synthesis of a large versican-like chondroitin sulfate proteoglycan by arterial smooth muscle cells. J Biol Chem. 1991;266(26):17640-17647.

33. Kiani C, Chen L, Wu YJ, Yee AJ, Yang BB. Structure and function of aggrecan. Cell Res. 2002;12(1):19-32.

34. Coucke PJ, et al. Mutations in the facilitative glucose transporter GLUT10 alter angiogenesis and cause arterial tortuosity syndrome. Nat Genet. 2006;38(4):452-457.

35. Tang PC, et al. Hyperplastic cellular remodeling of the media in ascending thoracic aortic aneurysms. Circulation. 2005;112(8):1098-1105.

36. Kimura N, et al. Gene expression profiling of acute type A aortic dissection combined with in vitro assessment. Eur J Cardiothorac Surg. 2017;52(4):810-817.

37. Erdheim J. Medionecrosis aortae idiopathica cystica. Virchows Arch Pathol Anat Physiol Klin Med. 1930;276(1):187-229.

38. Gutierrez PS, Reis MM, Higuchi ML, Aiello VD, Stolf NA, Lopes EA. Distribution of hyaluronan and dermatan/chondroitin sulfate proteoglycans in human aortic dissection. Connect Tissue Res. 1998;37(3-4):151-161.

39. Cattell MA, Hasleton PS, Anderson JC. Glycosaminoglycan content is increased in dissecting aneurysms of human thoracic aorta. Clin Chim Acta. 1994;226(1):29-46.

40. Oller J, et al. Nitric oxide mediates aortic disease in mice deficient in the metalloprotease Adamts1 and in a mouse model of Marfan syndrome. Nat Med. 2017;23(2):200-212.

41. Carrino DA, Arias JL, Caplan AI. A spectrophotometric modification of a sensitive densitometric Safranin O assay for glycosaminoglycans. Biochem Int. 1991;24(3):485-495.

42. Calabro A, Benavides M, Tammi M, Hascall VC, Midura RJ. Microanalysis of enzyme digests of hyaluronan and chondroitin/ dermatan sulfate by fluorophore-assisted carbohydrate electrophoresis (FACE). Glycobiology. 2000;10(3):273-281.

43. Calabro A, Midura R, Wang A, West L, Plaas A, Hascall VC. Fluorophore-assisted carbohydrate electrophoresis (FACE) of glycosaminoglycans. Osteoarthr Cartil. 2001;9 Supp1 A:S16-S22.

44. Foulcer SJ, et al. Determinants of versican-V1 proteoglycan processing by the metalloproteinase ADAMTS5. J Biol Chem. 2014;289(40):27859-27873.

45. Trapnell C, et al. Differential gene and transcript expression analysis of RNA-seq experiments with TopHat and Cufflinks. Nat Protoc. 2012;7(3):562-578. 